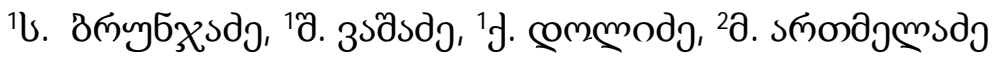

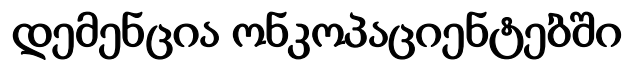

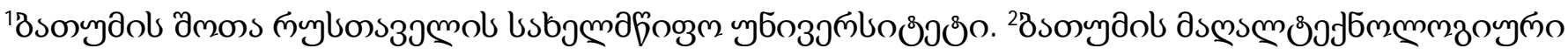

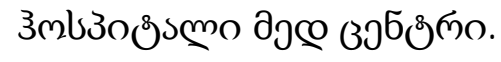

${ }^{1} \mathrm{~S}$. Brunjadze, ${ }^{1}$ Sh. Vashadze, ${ }^{1} \mathrm{~K}$. Dolidze ${ }^{2}$ M. Artmeladze

\section{Dementia in Oncological Patients}

\section{'Batumi Shota Rustaveli State University; 'Batumi hospital "M ed-Center".}

\section{Summary}

Oncological diseases are one of the main causes of morbidity and mortality in the world. The problem is quite topical since the number of patients with cancer is a sensitive theme. At present, there are about 20 million people having the disease all over the world. Hence, the number of elderly people with the disease is about 10-15\%. The purpose of our research was to study dementia in cancer patients. The study was conducted in the Batumi high-tech hospital (Batumi Oncological Center). 100 patients aged 25 to 75 participated in this study. A mong them, the 60 patients were female and 40 were male. At the same time, only 45 patients were operated on. Of these, 16 patients were operated after chemotherapy, 4 patients after radiotherapy and 35 patients during combination therapy. 6 patients with lung cancer, 22 patients with breast cancer, 6 patients with skin cancer, 7 patients with gastric cancer, 4 patients with bladder cancer, 15 patients with ovarian cancer, 25 patients with uterine cancer were examined. In the study, three of them had the family history of dementia. W e studied the patient's medical history, physiological data, assessed their mental and cognitive functions to determine the diagnosis of dementia. W e studied the patient's medical history, physiological data, assessed their mental and cognitive functions, mini-mental state examination, and carried out a survey of family members as the patients often had difficulty precisely describing the symptoms to determine the diagnosis of dementia. Out of 100 patients, $20 \%$ of them had mild dementia, $44 \%$ - moderate dementia and $35 \%$ - severe dementia. Among the patients examined, dementia was not observed in $1 \%$. Computed tomography of the brain was performed on the examined patients. Cerebral atrophy, a decrease in brain mass and volume in $34 \%$, cortical atrophy in $60 \%$ of patients and the expansion of

the ventricle system of the brain in $35 \%$ were revealed in the pathomorphological picture. The atrophic process is strongly expressed in the pariental (17\%), frontal and temporal lobes (76\%).

Therefore, dementia is quite common in cancer patients with severe and moderate depression, predominant in $79 \%$. Dementia was not observed only in one case, which is very important. The researchers point out reversible dementia after chemotherapy, which is reversible after a while.

Community involvement has great importance for the treatment and psychological adaptation to the new real ity of cancer patients. It is necessary to develop educational programs aimed at providing assistance from the moment of diagnosis to the end of treatment.

key words: Dementia and Cancer, Psychological therapies.

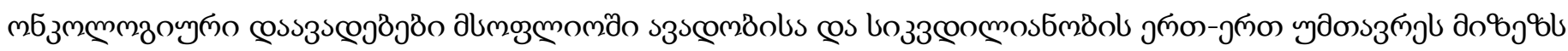

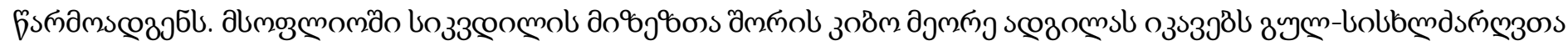

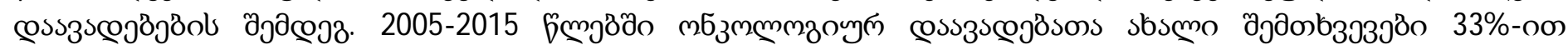

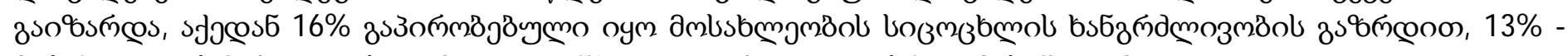

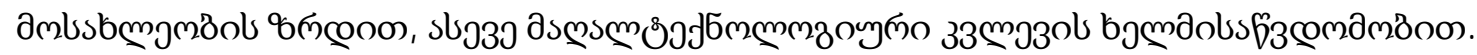

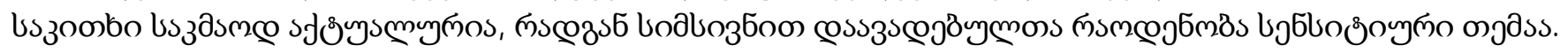

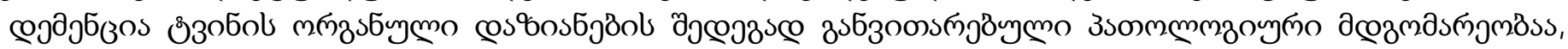

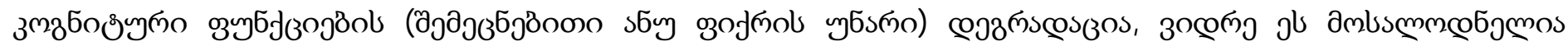




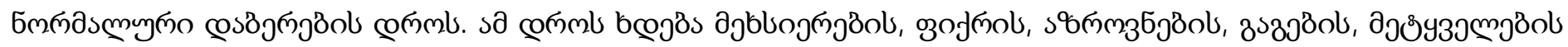

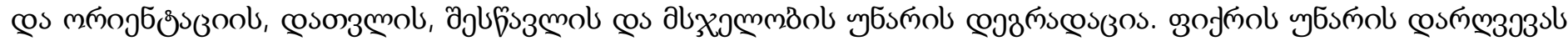

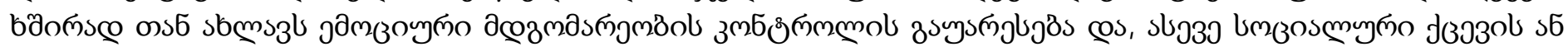

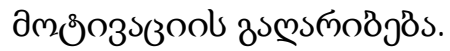

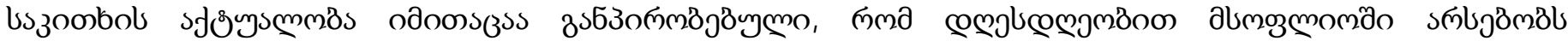

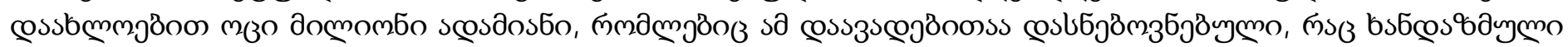

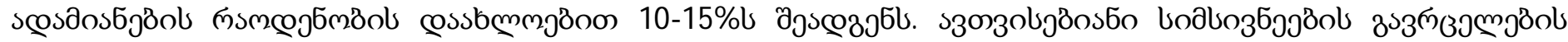

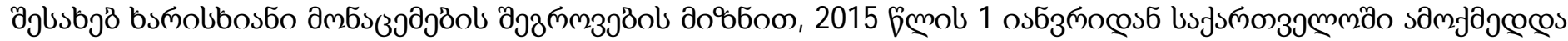

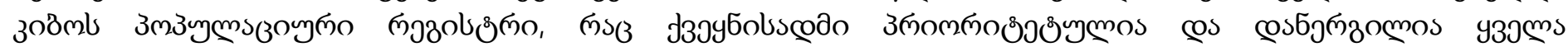

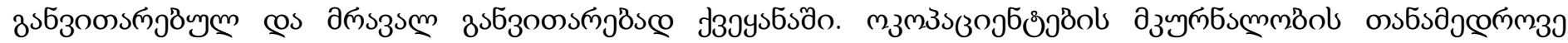

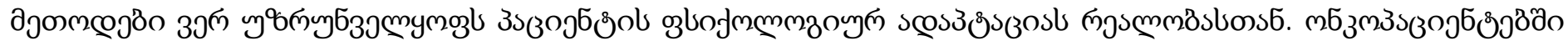

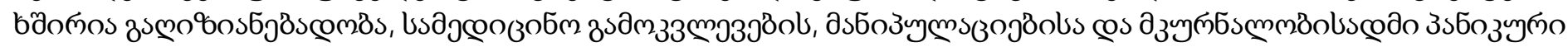

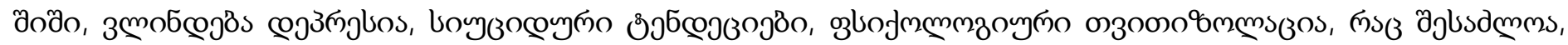

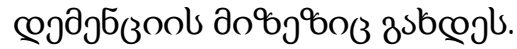

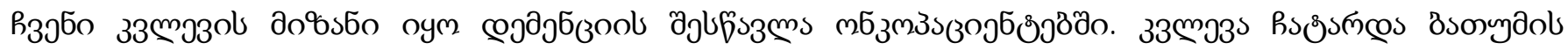

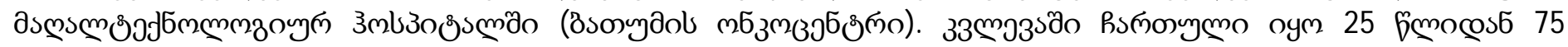

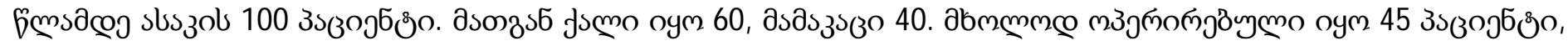

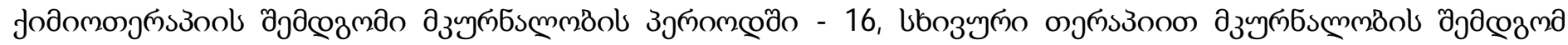

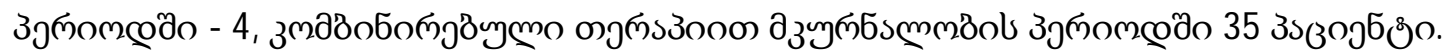

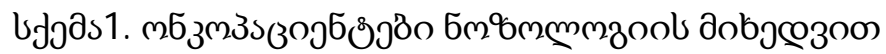

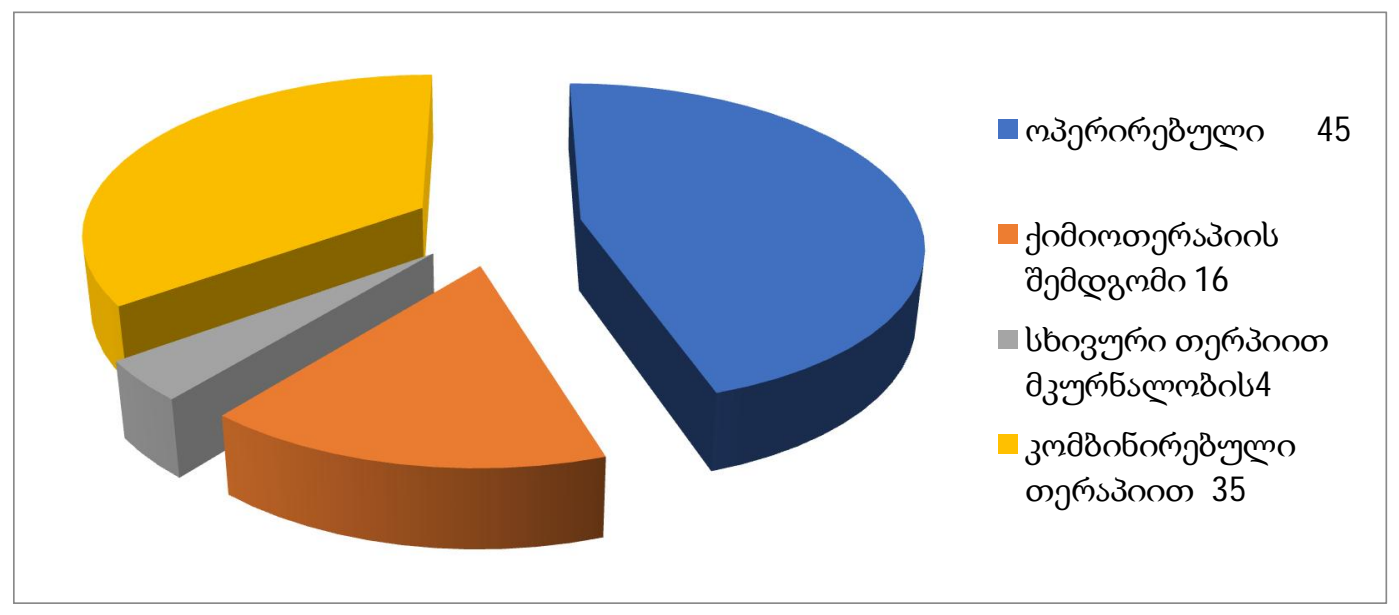

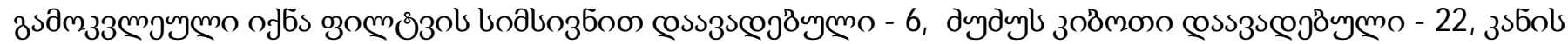

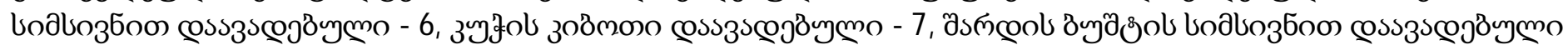

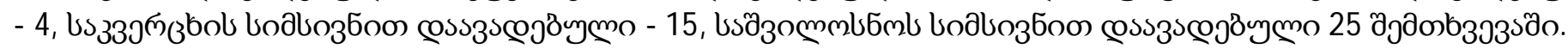




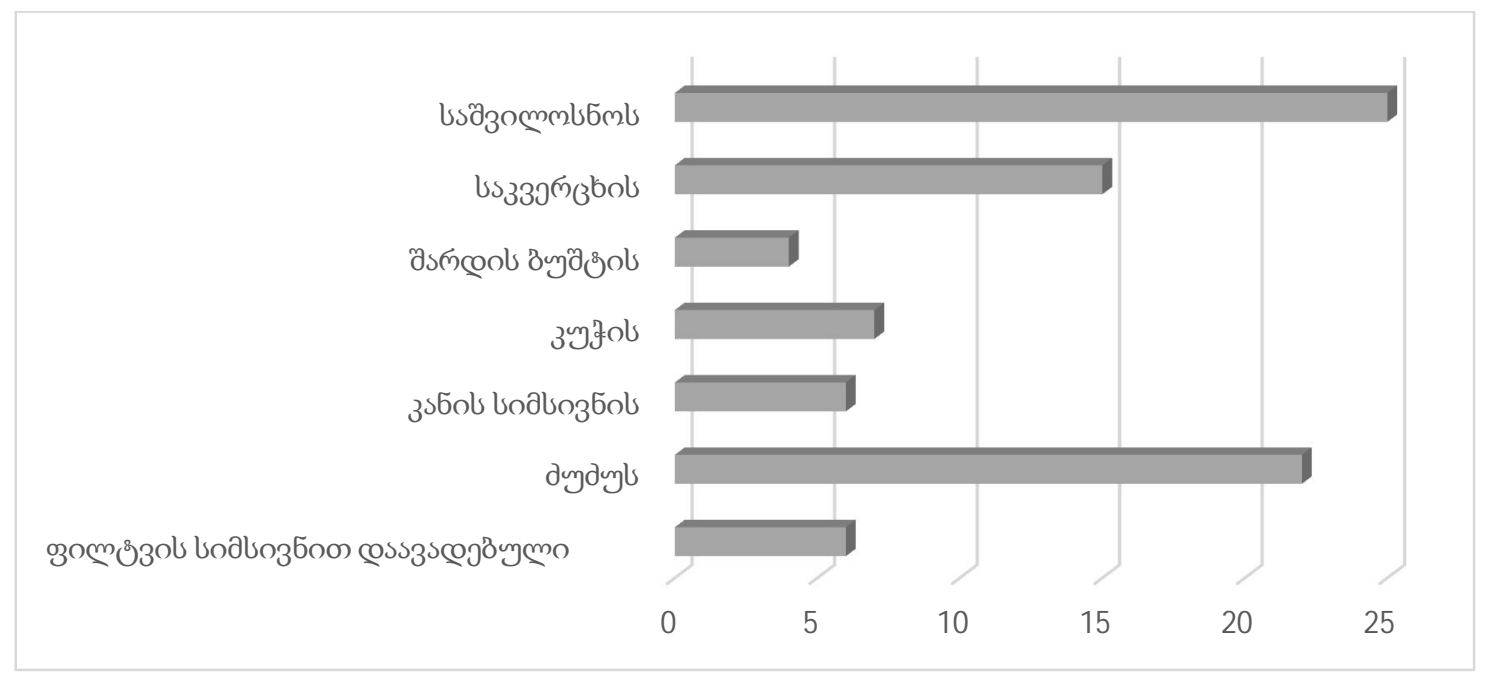

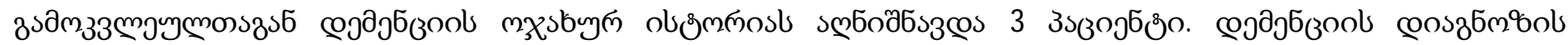

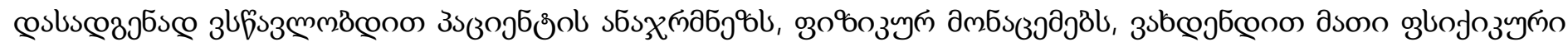

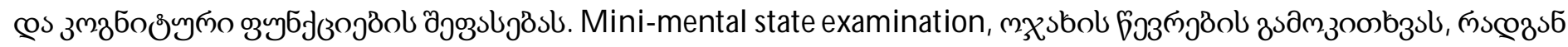

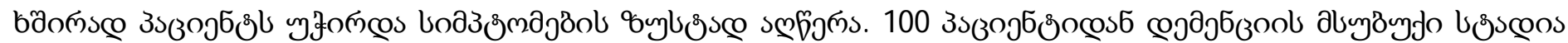

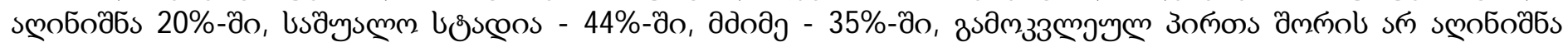
œэaj5

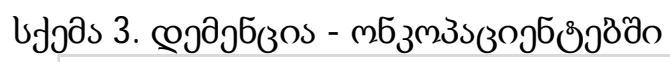

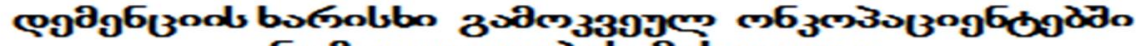

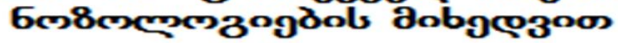

\begin{tabular}{|c|c|c|c|c|c|c|c|c|}
\hline & $\begin{array}{l}\text { gracts } \\
3 \text { ab }\end{array}$ & dydyls & 3.5eds & 3astols & 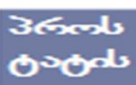 & 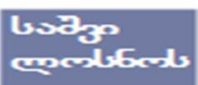 & $\begin{array}{l}\text { Dots } \\
\text { cols }\end{array}$ & $\begin{array}{l}\text { Wos30 } \\
\text { bols }\end{array}$ \\
\hline 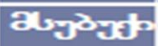 & 2 & 12 & o & o & o & 5 & I & o \\
\hline $\begin{array}{l}\text { Lays } \\
\text { cos }\end{array}$ & 2 & 6 & 2 & 5 & I & 15 & 2 & 10 \\
\hline बdरa & I & 6 & 4 & 2 & 5 & 5 & I & 5 \\
\hline 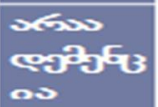 & I & o & o & o & o & o & o & o \\
\hline
\end{tabular}




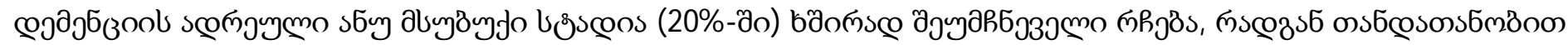

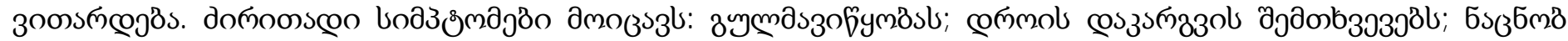

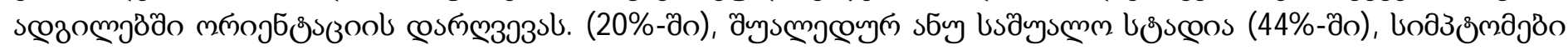

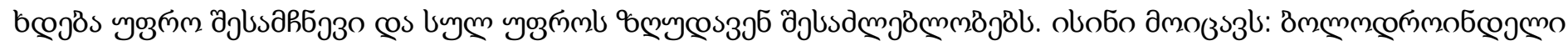

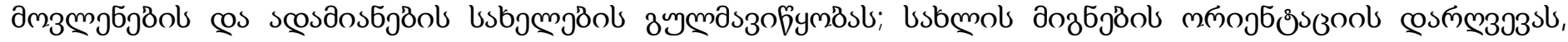

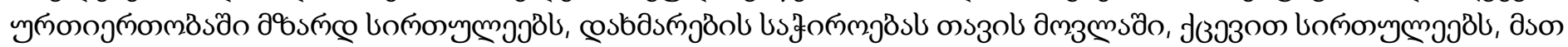

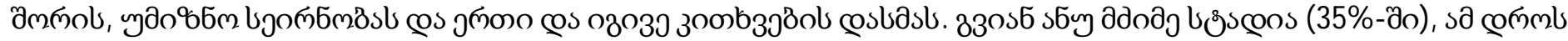

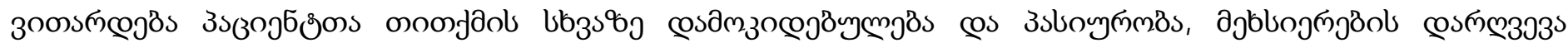

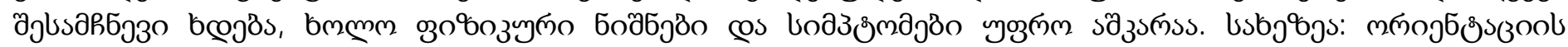

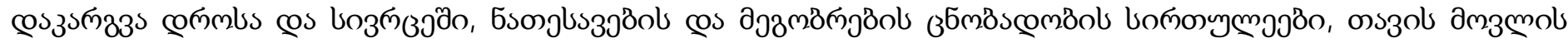

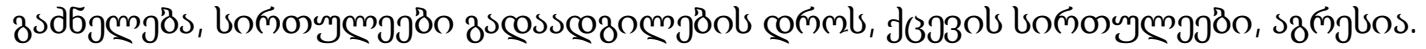

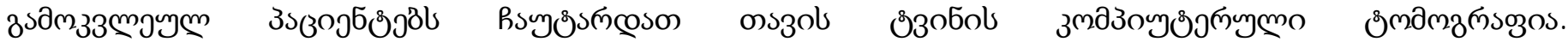

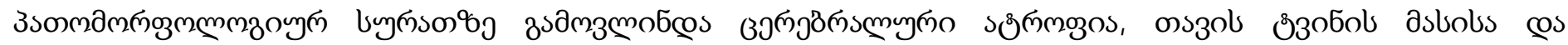

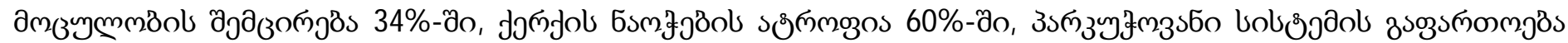

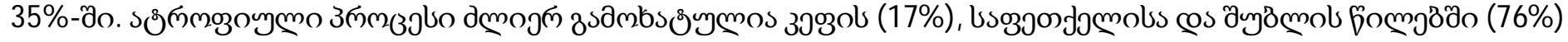

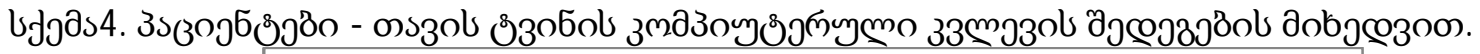

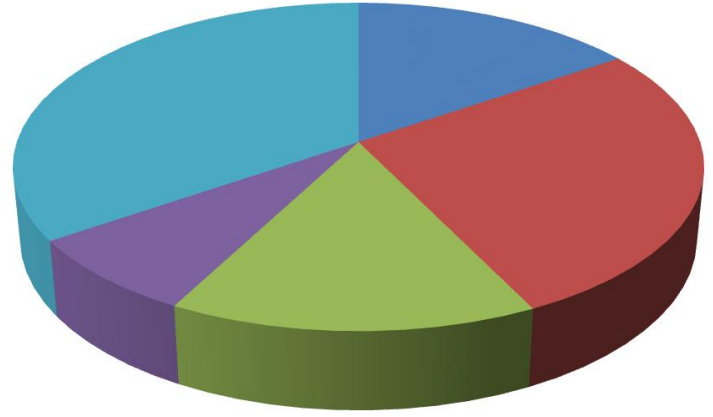

amßуmmònls

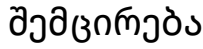

Jjmjols งষిmmoुns

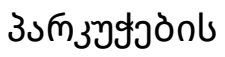

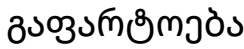

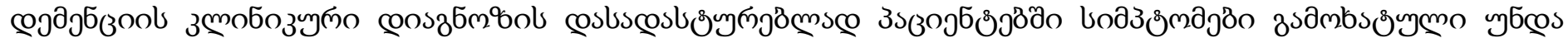

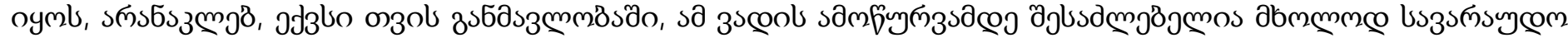

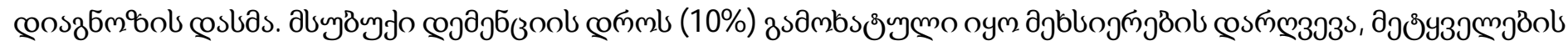

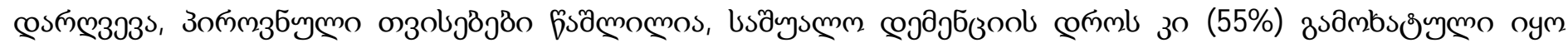

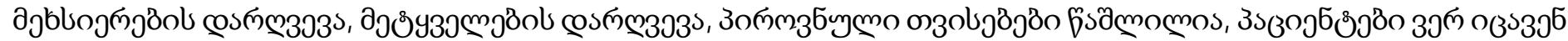

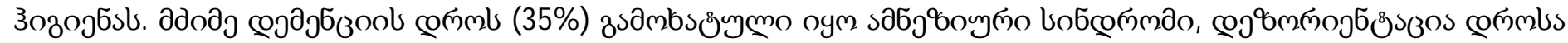

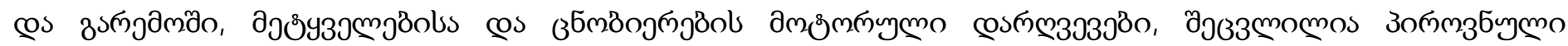

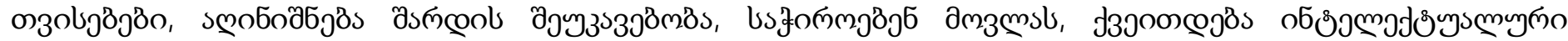

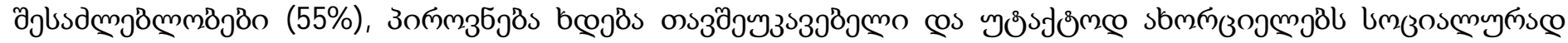

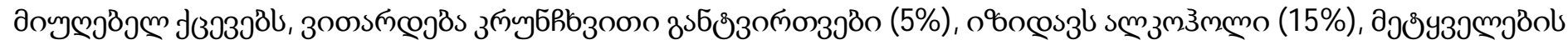

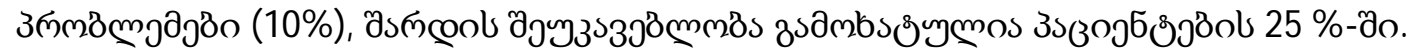

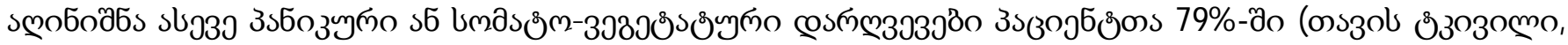

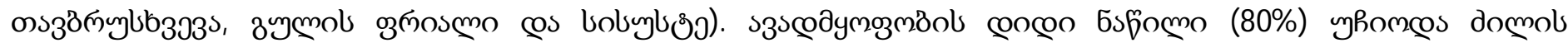

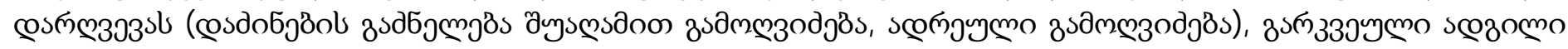

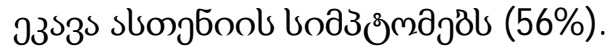




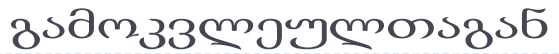

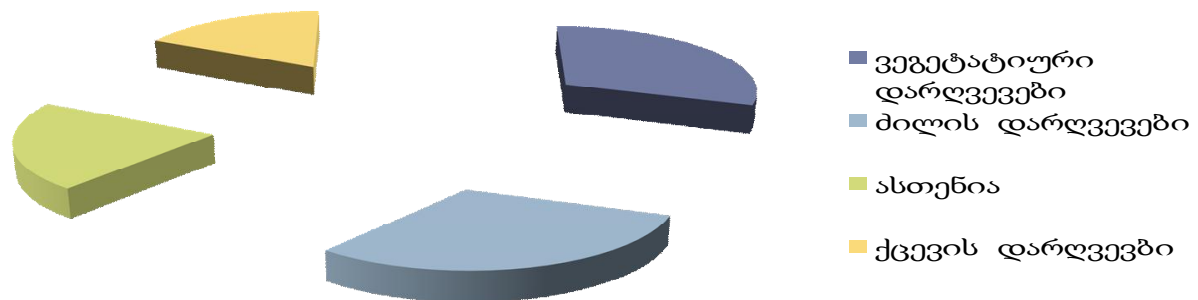

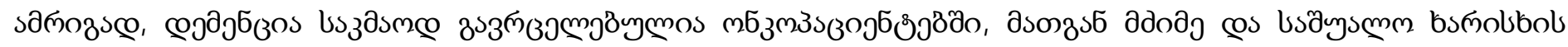

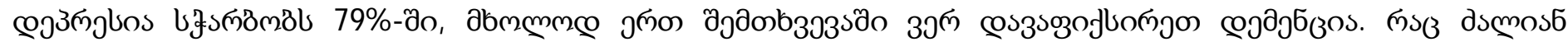

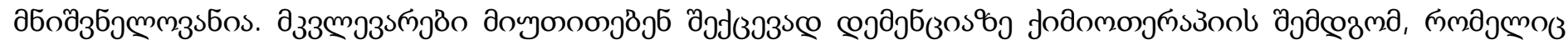

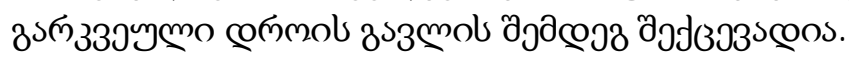

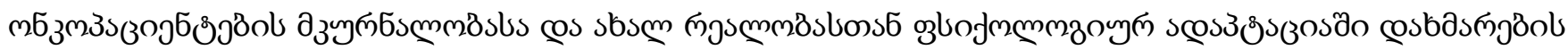

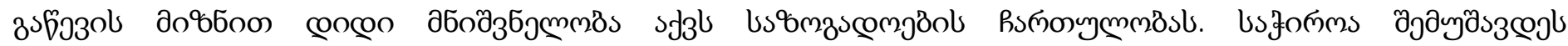

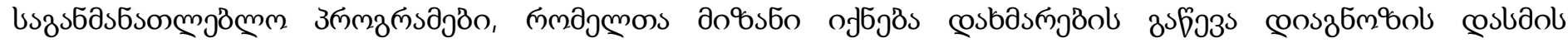

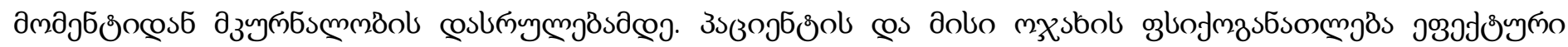

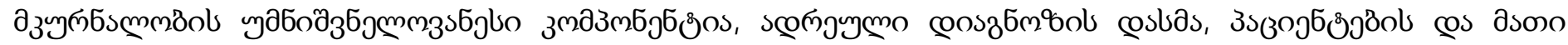

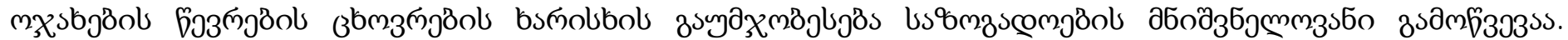

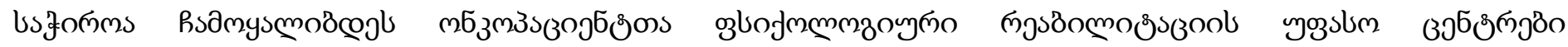

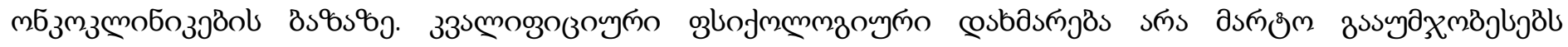

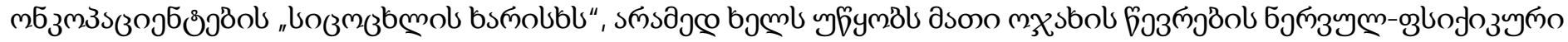

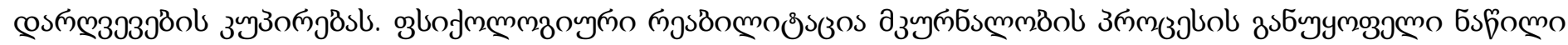

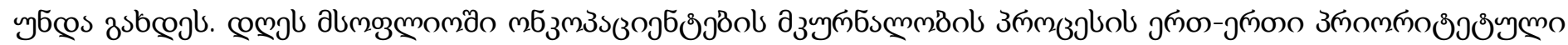

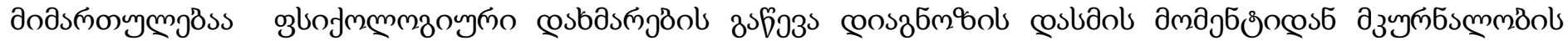

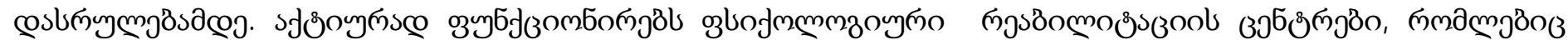
иsল

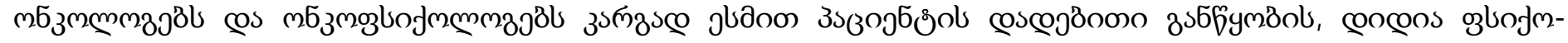

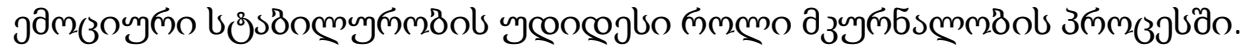

